\title{
A TYPE-2 FUZZY OPTIMIZATION MODEL FOR PROJECT PORTFOLIO SELECTION AND SCHEDULING BY INCORPORATING PROJECT INTERDEPENDENCY AND SPLITTING
}

\author{
Samaneh ZOLFAGHARI ${ }^{1}$, Seyed Meysam MOUSAVI ${ }^{1 *}$, \\ Jurgita ANTUCHEVIČIENÉ(D)2 \\ ${ }^{1}$ Department of Industrial Engineering, Shahed University, Tehran, Iran \\ ${ }^{2}$ Department of Construction Management and Real Estate, Faculty of Civil Engineering, Vilnius \\ Gediminas Technical University, Vilnius, Lithuania
}

Received 14 January 2021; accepted 25 March 2021

\begin{abstract}
This paper presents a new optimization model and a new interval type-2 fuzzy solution approach for project portfolio selection and scheduling (PPSS) problem, in which split of projects and re-execution are allowable. Afterward, the approach is realized as a multi-objective optimization that maximizes total benefits of projects concerning economic concepts by considering the interest rate and time value of money and minimizes the tardiness value and total number of interruptions of chosen projects. Besides, budget and resources limitation, newfound relations are proposed to consider dependency relationships via a synergy among projects to solve PPSS problem hiring interval type- 2 fuzzy sets. For validation of the model, numerical instances are provided and solved by a new extended procedure based on fuzzy optimistic and pessimistic viewpoints regarding several situations. In the end, their results are studied. The results show that it is more beneficial when projects are allowed to be split.
\end{abstract}

Keywords: project portfolio selection and scheduling, interdependent project, project splitting, interval type-2 fuzzy sets.

JEL Classification: C00, C52, D70, D81, G11, O22.

\section{Introduction}

Project portfolio selection and scheduling (PPSS) tries to regard proper candidates from a set of projects and make a thorough improvement scheme. Clearly, privileged project systems play an important role for organizations as imperfectly chosen projects most of the time do not work properly (Hall et al., 2015). Many project portfolio decision problems face uncertainty. Fuzzy sets theory is a common tool to handle non-probabilistic uncertainty in

*Corresponding author. E-mail: sm.mousavi@shahed.ac.ir

Copyright (c) 2021 The Author(s). Published by Vilnius Gediminas Technical University

This is an Open Access article distributed under the terms of the Creative Commons Attribution License (http://creativecommons. org/licenses/by/4.0/), which permits unrestricted use, distribution, and reproduction in any medium, provided the original author and source are credited. 
project management. Involving more uncertainty sources causes more complexity in modeling (Haghighi et al., 2019b; Peng \& Huang, 2020). Because of various kinds of complexities in reality, project managers cannot always define the exact membership function to show an uncertain boundary by type- 1 fuzzy sets (T1FSs). Therefore, type- 2 fuzzy set (T2FS) is used to pass on the vulnerability in the membership function and enables further degrees of freedom as compared to T1FS, by regarding remarkable engineering applications (e.g., Mirnezami et al., 2020; Mohagheghi et al., 2020; Haghighi et al., 2019a; Eshghi et al., 2019; Dorfeshan et al., 2019).

Chen and Askin (2009) presented a mixed-integer programming model aiming at maximizing economic issue by hiring an approach to deal with the problem. Liu and Wang (2011) proposed an approach to choose and schedule projects using constraint programming procedure with hiring time-dependent resource limitation. Huang and Zhao (2014) developed this issue and extended a genetic algorithm (GA) for solving it. Bhattacharyya et al. (2011) developed an optimization approach for the PPSS problem regarding benefits, costs, and risk. A developed model for the PPSS regarding divisibility and interdependency was proposed by Li et al. (2016). Dash et al. (2016) was the further instance that studied a MIP for the simultaneous PPSS problem considering resource constraints and interdependencies. Tofighian and Naderi (2015) used ant colony to decide the joint selection and scheduling problem. In their model, benefit and maximum levels of needed resources were optimized. The only type of interdependency modeled was mutual exclusiveness. Watermeyer and Zimmermann (2020) considered partially renewable resources and general temporal constraints for the RSPSP problem. They developed a branch-and-bound method to solve the model with the aim of project duration minimization. Egri and Kis (2020) studied material allocation for scheduling of the projects with competing self-interested agents. They assumed the different supplying dates for required materials. Rahman et al. (2020) extended a genetic algorithmbased memetic algorithm to deal with RCPSP problems. They improved the deficiency of randomness of genetic algorithm by applying different heuristics. Relich (2021) developed a decision support system for NPD project portfolio selection. Resource allocating, evaluating input and output dependency, and forecasting an output variable comprised the structure of system. Nowak and Trzaskalik (2021) extended a stochastic discrete multi-objective model for project portfolio selection. They solved a model by an interactive method by employing trade-of analysis. Salehi (2018) solved the PPSS under fuzzy conditions by the AWFP procedure. Zhang et al. (2019) developed a multi-objective evolutionary procedure for the PPSS regarding fuzzy conditions. Dixit and Tiwari (2020) proposed a PPSS in terms of risk measure.

According to the vagueness and uncertainties in the project management, fuzzy sets theory has been used widely in this field. Wang and Hwang (2007) developed a fuzzy zeroone integer programming approach to identify optimum R\&D project portfolio. Liao and Ho (2010) presented a fuzzy binomial method to assess a project and extended a proper approach to calculate the mean value of fuzzy NPV. Mohagheghi et al. (2015) optimized the PPSS and considered net cash flows as IVF-numbers. Damghani et al. (2011) proposed a two-stage decision-making model. In their modeling, fuzzy numbers were hired to represent the values corresponding with the projects and resources.

For multi-period models, most available studies presume that each nomination project cannot be divided into a number of sub-parts for various periods; notwithstanding, numer- 
ous pragmatic constraints, e.g., capital, scarcity of labor, project difficulties, and unpredicted events may provoke unavoidable interferences for projects (Baker \& English, 2011). As a result, joining interferences to the project is a characteristic call from practice. Therefore, $\mathrm{Li}$ et al. (2015) brought up the issue of project divisibility into the PPSS.

Referring to papers studied the PPSS problems, a number of research gaps are highlighted. Many of the researches aforesaid looked the issue of the PPSS without any discontinuity in ongoing. Although a case is discussed, in which projects could be split if it is needed, mostly when the amount of resources and budgets are not sufficient and restarted later.

When more than one interdependent projects are selected and run simultaneously, a firm may get an advantage more (or not exactly) the basic amount of the benefits from every single undertaking. The occasion is entitled interdependency between the ventures (Liesiö et al., 2008). The extra advantage over the amount of single advantages is entitled to the synergistic advantage. Distinguishing and demonstrating the interdependency among projects may make substantial reserve funds and advantages to the client (Santhanam \& Kyparisis, 1996), while the piece of benefit not exactly the amount of single advantage is entitled the serious misfortune (Fox et al., 1984). A few examinations on PPSP have as often as possible emphasized the criticalness and need of broadening optimization approaches that mean advantages and misfortunes from the interdependency among projects (Shafahi \& Haghani, 2013; Tao \& Schonfeld, 2006).

According to the project portfolio selection and scheduling studies, many researchers neglected the interruption of projects as common event in project management, specifically having resource limitation. Moreover, more research considered interdependency as a group of projects should be selected simultaneously or, in contrast, should not be selected concurrently. Therefore, interdependency considering as positive or negative synergy with their effects on resource usage is still scarce. Taking into account both splitting and interdependency in terms of synergy not only leads to more profitable project portfolio selection and scheduling but also is close to real cases. Complexity makes usage of uncertain information unavoidable. T2FSs help managers to make a better decision by providing them more flexibility in the decision-making process. There are no investigation efforts on project portfolio selection and scheduling to the best of our knowledge, considering splitting and synergy simultaneously with T2FSs under a multi-objective model.

This paper presents the PPSSP under the projects splitting conditions; Proposed study develops this class of interdependency with regard to a synergy between performed projects in each time period and formulizes a $0-1$ integer model for the problem. In developed study, a linear multi-objective model which jointly optimizes the PPSS is proposed. Presented model is subject to general conditions making it enforceable to the real environment. The problem objective aims at maximizing the total net present value from the portfolio. The projects benefit depends on the time that projects are selected. Taking into consideration the split of projects to discontinue the projects and launch another project because of resource scarcity. The minimization of the number of splitting is another objective. To prevent the high lateness of selected project, the total tardiness values for each project is minimized.

In addition, in this work, a novel fuzzy solution method is presented based on optimistic and pessimistic standpoint to deal with a new fuzzy linear programming problem with ambiguity uncertainty. The whole coefficients of the problem are IT2F triangular numbers. 
The proposed method solves a model three times to find an optimal solution. At the first step, the optimistic model is constructed while all IT2F-triangular parameters stand on the best corresponding value. Therefore, the multi-objective crisp model is obtained. The multiobjective model is transformed to the single objective model, upon the current literature approach, and optimized. At the second step, the aforementioned procedure is applied, but all IT2F-triangular parameters take their worst values as a pessimistic model. Two solutions are achieved based on optimistic and pessimistic procedure. The final optimal solution is obtained based on both optimistic and pessimistic values. In the third step, a new model is created by the concept of membership function. The objective function is maximized the value of membership function subject to constraints. The constraints with IT2F-triangular parameters are regarded with discounting the entirely optimistic viewpoint. The final compromise solution is benefited both optimistic and pessimistic standpoints.

The rest of the study is organized as follows: Section 1 reviews the theory of T2FSs. Section 2 defines the PPSS problem and presents a new optimization model. Section 3 provides a new T2F solution methodology. Sections 4 and 5 regard practical instances and computational processes. Conclusions are given in last section.

\section{Type-2 Fuzzy sets}

To better understanding, a brief explanation of interval type-2 fuzzy triangular sets (IT2FTSs) can be given.

Definition 1. T2Fs have membership grades that are specified by ITFSs. $\tilde{A}$ also can be proposed as demonstrated in the following (Mendel et al., 2006):

$$
\tilde{A}=\int_{x \in X} \int_{u \in J_{x}} \frac{\mu_{\tilde{A}}(x, \mu)}{(x, \mu)} \text {, where union over all admissible } x \text { and } u \text { are represented. }
$$

Definition 2. If in $\tilde{A}$ all $\mu_{\tilde{A}}(x, \mu)=1$, then $\tilde{A}$ is an IT2FS. This set is considered as a particular instance of a type-2 fuzzy set. The following shows this set $\tilde{A}=\int_{x \in X} \int_{u \in J_{x}} \frac{1}{(x, \mu)}$,
where $J_{x} \subseteq[0,1]$.

Definition 3. A bounded region describes the initial membership uncertainty in a T2FS. This region called the footprint of uncertainty, where $F O U=\cup_{x \in X} J_{x}$. The FOU consists two membership functions (MFs), a lower MF (LMF), $\underline{\mu}_{\tilde{A}}$, and upper MF (UMF), $\bar{\mu}_{\tilde{A}}$. Therefore, an IT2FS is composed by two type- 1 fuzzy sets.

Definition 4. The core of the IT2F number $\tilde{A}=[\underline{A}, \bar{A}]$ is $\operatorname{Core}(\tilde{A})=[\min \operatorname{Core}(\underline{A})$, $\max \operatorname{Core}(\underline{A})]$ when $\varnothing \neq \operatorname{Core}(\underline{A}) \subseteq \operatorname{Core}(\bar{A})$, where $\varnothing$ is the empty set and lower and upper MFs of $\tilde{A}$ defined by $\underline{\mu}_{\tilde{A}}$ and $\bar{\mu}_{\tilde{A}}$, respectively (Javanmard and Nehi, 2019).

Definition 5. IT2FT number, $\tilde{A}$ as depicted in Figure 1 is characterized on interval $\left[\bar{a}^{l}, \bar{a}^{u}\right]$, its lower MF and upper MF are equal to $\underline{h} \in[0,1]$ in $\underline{a}$ and $\bar{h} \in[0,1]$ in $\bar{a}$, respectively, where $\bar{a}^{l}<\underline{a}^{l}<m<\underline{a}^{u}<\bar{a}^{u}$ and $\bar{a}^{l}<m<\bar{a}^{u}$. So, the IT2FT number, $\tilde{A}$ is denoted by $\tilde{A}=(\underline{A}, \bar{A})=\left(\left(\underline{a}^{l}, m, \underline{a}^{u}\right),\left(\bar{a}^{l}, m, \bar{a}^{u}\right)\right)($ Javanmard \& Nehi, 2019). 


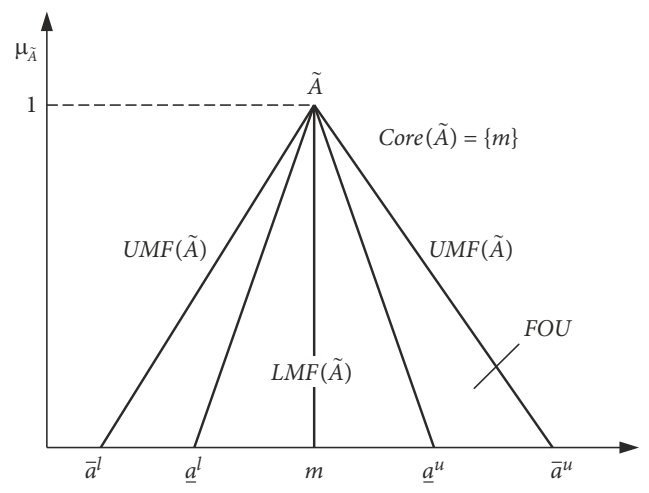

Figure 1. IT2FT number

\section{Problem description and formulation}

\subsection{Problem description}

Presume an organization that is based on projects can have $N$ recommended candidates, any of them can have its profitability, and a group of projects have interdependency. Concerning selecting the portfolio, the purpose can choose top candidates based on the aims as well as available constraints. We can define that candidate in which period is picked and accomplished. Presume that the firm can assess candidates considering a class of evaluation factors, which in period $k$ taking on the particular time frame each chosen candidate is in. The firm can have determined several subsets of candidates $A j$ such that if in period $k$ the portfolio involves various candidates.

Traditionally, most academics presume that once a project begins, it must be executed without interruption. Although, in real-world cases, interruption is a repeated incidence in the project execution, splitting is a helpful managerial technique that can advance pay and influence the productivity of corporate. As a result, this study will be most interested in project splitting when selecting a project portfolio. To handle incomplete information, uncertainty theory is hired. Utilizing uncertainty theory can manage imprecise assessments. As a result, this theory could be applied to build proposed optimization approach more realistic.

Objective functions in the optimization approach include: 1 . Selected projects expected profit maximization; 2 . The lateness of selected projects minimization; and 3 . Total number of interruptions of selected projects minimization.

\subsection{Parameters and model}

Sets and indices

$$
\begin{aligned}
& \quad i=\{1, \ldots, m\}-\text { Set of projects } \\
& t=\{1, \ldots T+1\}-\text { Set of time periods } \\
& k=\{1, \ldots, K\}-\text { Set of resources } \\
& s=\{1, \ldots, S\}-\text { Set of synergies } \\
& \qquad A_{s}-\text { Set of projects associated with synergy } s
\end{aligned}
$$




\section{Parameters}

$\tilde{d}_{i}$ - Duration time for project $i$

$\tilde{r}_{i k t}$ - Resource $k$ requirement of project $i$ at time $t$

$\widetilde{E}_{s k}$ - Amount of decreasing resource usage $k$ under synergy $s$

$\tilde{C}_{k t}$ - Cost of per resource $k$ at time $t$

$r$ - Interest rate

$B$ - Total budget

$\widetilde{R R}_{k t}$ - Available resource $k$ at time $t$

$L_{s}$ - Minimum number of executing projects belong to set $A_{s}$ in order to occurring synergy $s$

$U_{s}$ - Maximum number of executing projects belong to set $A_{s}$ in order to occurring synergy $s$ $D D_{i}$ - Due date of project $i$

$\tilde{P}_{i t}-$ Expected profit of project $i$ when is selected at time $t$

$B N$ - Big number

$N T$ - Last time of planning horizon equal to $T$

\section{Decision variables}

$x_{i t}$ - Taking value 1 if project $i$ is chosen at time $t, 0$ otherwise

$y_{i t}-$ Taking value 1 if project $i$ is run at time $t, 0$ otherwise

$z_{s t}$ - Taking value 1 if synergy $s$ is occurred at time $t, 0$ otherwise

$w_{i t}$ - Taking value 1 if project $i$ is performed at time $t$ and $t+1$

$v_{i t}$ - Last executed period of project $i$ at time $t$

$L S_{i}$ - Lateness of project $i$

Mathematical formulation

$$
\begin{aligned}
& \min z_{1}=\sum_{i=1}^{m} L S_{i} \\
& \max z_{2}=\sum_{i=1}^{m} \sum_{t=1}^{T-1} \tilde{P}_{i t}(1+r)^{-(t+1)} y_{i t}-\sum_{i=1}^{m} \sum_{k=1}^{K} \sum_{t=1}^{T} \tilde{i}_{i k t} \tilde{C}_{k t}(1+r)^{-t} y_{i t}+ \\
& \sum_{k=1}^{K} \sum_{s=1}^{S} \sum_{t=1}^{T} \tilde{E}_{s k} \tilde{C}_{k t} z_{s t}(1+r)^{-t} \\
& \min z_{3}=\sum_{i=1}^{m} \sum_{t=1}^{T} y_{i t}-w_{i(t+1)}
\end{aligned}
$$

s.t.

$$
\begin{aligned}
& \sum_{t=1}^{T} x_{i t} \leq 1, \quad \forall i=1, \ldots m ; \\
& \sum_{t=1}^{T}\left(t+\tilde{d}_{i}\right) x_{i t} \leq T, \quad \forall i=1, \ldots m ; \\
& \sum_{t^{\prime}=t}^{T} y_{i t^{\prime}}-\tilde{d}_{i} x_{i t} \geq 0, \quad \forall i=1, \ldots m, t=1, \ldots, T-\tilde{d}_{i}+1 ;
\end{aligned}
$$




$$
\begin{aligned}
& \sum_{i=1}^{m} \sum_{k=1}^{K} \sum_{t=1}^{T} \tilde{r}_{i k t} \tilde{C}_{k t}(1+r)^{-t} y_{i t}+\sum_{k=1}^{K} \sum_{s=1}^{S} \sum_{t=1}^{T} \tilde{E}_{s k} \tilde{C}_{k t} z_{s t}(1+r)^{-t} \leq B ; \\
& \sum_{i=1}^{m} \tilde{r}_{i k t} y_{i t}+\sum_{s=1}^{S} \tilde{E}_{s k} z_{s t} \leq \widetilde{R R} k t, \forall t=1, \ldots T, k=1, \ldots, K ; \\
& L_{s} z_{s t} \leq \sum_{i \in A_{s}} y_{i t}, \forall t=1, \ldots, T, s=1, \ldots, S ; \\
& \sum_{i \in A_{s}} y_{i t} \leq U_{s} z_{s t}+\left(L_{s}-1\right), \forall t=1, \ldots, T, s=1, \ldots, S ; \\
& \tilde{d}_{i} \sum_{t=1}^{T} x_{i t}=\sum_{t=1}^{T} y_{i t}, \forall i=1, \ldots, m ; \\
& t^{\prime} y_{i t^{\prime}} \leq v_{i t}, \forall i=1, \ldots, m, t=2, \ldots, T+1, t^{\prime}=1, \ldots, t-1 ; \\
& v_{i t} \leq t^{\prime}\left(y_{i\left(t^{\prime}+1\right)}+1-w_{i\left(t^{\prime}+1\right)}\right)-B N\left(y_{i t^{\prime}}-1-\sum_{l=t^{\prime}+1}^{t-1} y_{i l}\right) \\
& \forall i=1, \ldots, m, t=2, \ldots, T+1, t^{\prime}=1, \ldots, t-1 ; \\
& y_{i(t+1)} \leq y_{i(t+1)}, \forall i=1, \ldots, m, t=1, \ldots, T ; \\
& w_{i(t+1)} \leq y_{i t}, \forall i=1, \ldots, m, t=1, \ldots, T ; \\
& y_{i(t+1)}+y_{i t} \leq w_{i(t+1)}+1, \forall i=1, \ldots, m, t=1, \ldots, T ; \\
& S_{i} \geq v_{i N T}, w_{i t}, z_{s t} \in\{0,1\}, v_{i t} \geq 0 . \\
& w_{i=1} x_{i t}, \forall i=1, \ldots, m ;
\end{aligned}
$$

The first objective function, Eq. (1), aims at tardiness value minimization. The second objective function, Eq. (2), aims at total profit of chosen and executed candidates maximization with respect to the time value of money. Eq. (3) minimizes the total amount of interruption in performing chosen candidates. Relation (4) guarantees that the candidates can be chosen in at most one period. Relation (5) represents that candidates are performed during planning horizon. Relation (6) makes sure that candidates are executed discontinuously. Relation (7) indicates that total cost could not be larger than the budget. Eq. (8) guarantees that chosen candidates can be planned considering resource limitations in every period. Eqs (9) and (10) make sure that the minimum and maximum numbers of executing projects for an interdependent subset have been satisfied in each period, and the synergy occurs. Relation (11) assures that the chosen candidates could be performed according to duration time. Relations (12) and (13) calculate the final performing period for candidate $i$ before period $t$. Eq. (14) computes the tardiness values for each project. Eqs (15)-(17) calculates the value of a Boolean variable, $w_{i t}$, is 1 if and only if several proportions of candidate $i$ can be consecutively performed in both periods $t$ and $t+1$. 


\section{Solution methodology}

In this part, a new procedure is presented based on the recent literature (Selim \& Ozkarahan, 2008; Javanmard \& Nehi, 2019; Kundu et al., 2019) for solving IT2FT-problem where the entire of coefficients is IT2FT-number. Take into account the IT2FT-linear programming problem where right-hand sides of the constraints, objective functions, and constraints coefficients are uncertain as given below:

$$
\operatorname{Max} z_{1}=\sum_{j=1}^{n} \tilde{c}_{j} x_{j}, \operatorname{Min} z_{2}=\sum_{j=1}^{n} \tilde{f}_{j} x_{j}
$$

s.t.

$$
\begin{aligned}
& \sum_{j=1}^{n} \tilde{a}_{i j} x_{j} \leq \tilde{b}_{i}, \quad i=1, \ldots, m ; \\
& \sum_{j=1}^{n} \tilde{d}_{k j} x_{j} \leq \tilde{e}_{k}, \quad k=1, \ldots, K ; \\
& x_{j} \geq 0, \quad j=1, \ldots, n,
\end{aligned}
$$

where $\tilde{c}_{j}, \tilde{a}_{i j}, \tilde{b}_{i}, \tilde{d}_{k j}$ and $\tilde{e}_{k}$ are IT2FT-numbers denoted by $\tilde{c}_{j} \in\left[\underline{c}_{j}, \bar{c}_{j}\right], \tilde{a}_{i j} \in\left[\underline{a}_{i j}, \bar{a}_{i j}\right]$, $\tilde{b}_{i} \in\left[\underline{b}_{i}, \bar{b}_{i}\right], \tilde{d}_{k j} \in\left[\underline{d}_{k j}, \bar{d}_{k j}\right], \tilde{f}_{j} \in\left[\underline{f}_{j}, \bar{f}_{j}\right]$ and $\tilde{e}_{k} \in\left[\underline{e}_{k}, \bar{e}_{k}\right]$.

Step 1. Create the model taking into consideration the optimistic viewpoint for the model (19) below:

$$
\begin{aligned}
& \operatorname{Max} z_{1}=\sum_{j=1}^{n} \bar{c}_{j}^{l} x_{j}, \quad \operatorname{Min} z_{2}=\sum_{j=1}^{n} \bar{f}_{j}^{l} x_{j} \\
& \text { s.t. } \\
& \qquad \sum_{j=1}^{n} \bar{a}_{i j}^{l} x_{j} \leq \bar{b}_{i}^{u}, \quad i=1, \ldots, m ; \\
& \quad \sum_{j=1}^{n} \bar{d}_{k j}^{u} x_{j} \geq \bar{e}_{k}^{l}, \quad k=1, \ldots, K, \\
& \quad x_{j} \geq 0, \quad j=1, \ldots, n .
\end{aligned}
$$

Now, the multi-objective deterministic model (20) is obtained. The multi-objective model can be converted to a single-objective model as follows:

$$
\begin{aligned}
& \operatorname{Max} z=\gamma \lambda+(1-\gamma) \sum_{l=1}^{2} \delta_{l} \lambda_{l} \\
& \text { s.t. } \\
& \quad \sum_{j=1}^{n} \bar{a}_{i j}^{l} x_{j} \leq \bar{b}_{i}^{u}, \quad i=1, \ldots, m ; \\
& \quad \sum_{j=1}^{n} \bar{d}_{k j}^{u} x_{j} \geq \bar{e}_{k}^{l}, \quad k=1, \ldots, K ;
\end{aligned}
$$




$$
\begin{aligned}
& \lambda+\lambda_{l} \leq \mu_{i}(x), \quad l=1,2 ; \\
& \lambda, \lambda_{l} \in[0,1], \quad l=1,2 ; \\
& x_{j} \geq 0, \quad j=1, \ldots, n,
\end{aligned}
$$

where $\gamma$ and $\delta_{l}$ belong to $[0,1]$ and $\sum_{l=1}^{2} \delta_{l}=1$ which determined by decision maker. $\mu_{1}(x)=\frac{z_{1}-z_{1}^{N I S}}{z_{1}^{P I S}-z_{1}^{N I S}}$ and $\mu_{2}(x)=\frac{z_{2}^{N I S}-z_{2}}{z_{2}^{N I S}-z_{2}^{P I S}}$ are for maximization and minimization objective functions, respectively. We have:

$$
\begin{aligned}
& z_{1}^{\text {PIS }}=\max z_{1}, \quad z_{2}^{P I S}=\min z_{2}, \\
& z_{1}^{\text {NIS }}=\min z_{1}, \quad z_{2}^{\text {NIS }}=\max z_{2} .
\end{aligned}
$$

Now solve the model (21) and called $\operatorname{Max} z=z^{\prime}$.

Step 2. Creating the problem model (22) by supposing the pessimistic standpoint of model (19) below:

$$
\operatorname{Max} z_{1}=\sum_{j=1}^{n} \bar{c}_{j}^{l} x_{j}, \operatorname{Min} z_{2}=\sum_{j=1}^{n} \bar{f}_{j}^{u} x_{j}
$$

s.t.

$$
\begin{aligned}
& \sum_{j=1}^{n} \bar{a}_{i j}^{u} x_{j} \leq \bar{b}_{i}^{l}, \quad i=1, \ldots, m ; \\
& \sum_{j=1}^{n} \bar{d}_{k j}^{l} x_{j} \geq \bar{e}_{k}^{u}, \quad k=1, \ldots, K ; \\
& x_{j} \geq 0, \quad j=1, \ldots, n .
\end{aligned}
$$

The crisp single objective model can be created as:

$$
\operatorname{Max} z=\gamma \lambda+(1-\gamma) \sum_{l=1}^{2} \delta_{l} \lambda_{l}
$$

s.t.

$$
\begin{aligned}
& \sum_{j=1}^{n} \bar{a}_{i j}^{u} x_{j} \leq \bar{b}_{i}^{l}, \quad i=1, \ldots, m ; \\
& \sum_{j=1}^{n} \bar{d}_{k j}^{l} x_{j} \geq \bar{e}_{k}^{u}, \quad k=1, \ldots, K ; \\
& \lambda+\lambda_{l} \leq \mu_{i}(x), \quad l=1,2 ; \\
& \lambda, \lambda_{l} \in[0,1], \quad l=1,2 ; \\
& x_{j} \geq 0 ; \quad j=1, \ldots, n .
\end{aligned}
$$

Solving model (23), we have $\operatorname{Max} z=z^{\prime \prime}$. 
Step 3. Let us define $\operatorname{Max}\left(z^{\prime}, z^{\prime \prime}\right)=z_{\max }$ and $\operatorname{Min}\left(z^{\prime}, z^{\prime \prime}\right)=z_{\min }$.

The below membership function, for the maximization problem, is defined to figure out an optimized compromised solution.

$$
\mu(z)=\frac{z-z_{\min }}{z_{\max }-z_{\min }} .
$$

As a result, maximum $\mu(Z)$ conveys a preferable solution for the problem of maximization. Here to procure the optimized compromise solution, make sure maximum possible satisfaction of constraints, note an auxiliary variable $\varphi(0 \leq \varphi \leq 1)$ and develop the below programming model, $z \geq z_{\min }+\varphi\left(z_{\max }-z_{\min }\right)$ :

$\operatorname{Max} \varphi$

s.t.

$$
\begin{aligned}
& \mu(z) \geq \varphi ; \\
& \sum_{j=1}^{n} a_{i j}^{l} x_{j} \leq \underline{b}_{i}^{u}, \quad i=1, \ldots, m ; \\
& \sum_{j=1}^{n} d_{k j}^{u} x_{j} \geq \underline{e_{k}^{l}, \quad k=1, \ldots, K ;} \\
& \lambda+\lambda_{l} \leq \mu_{i}(x), \quad l=1,2 ; \\
& \lambda, \lambda_{l} \in[0,1], \quad l=1,2 ; \\
& x_{j} \geq 0 ; \quad j=1, \ldots, n .
\end{aligned}
$$

The model (24) is constructed neither completely optimistic nor pessimistic. A semioptimistic standpoint is applied. We procure a compromise solution that occurs in the range of $z_{\min }$ and $z_{\max }$, after solving the problem (24).

Figure 2 depicts the brief schematic overview of the aforementioned solution methodology.

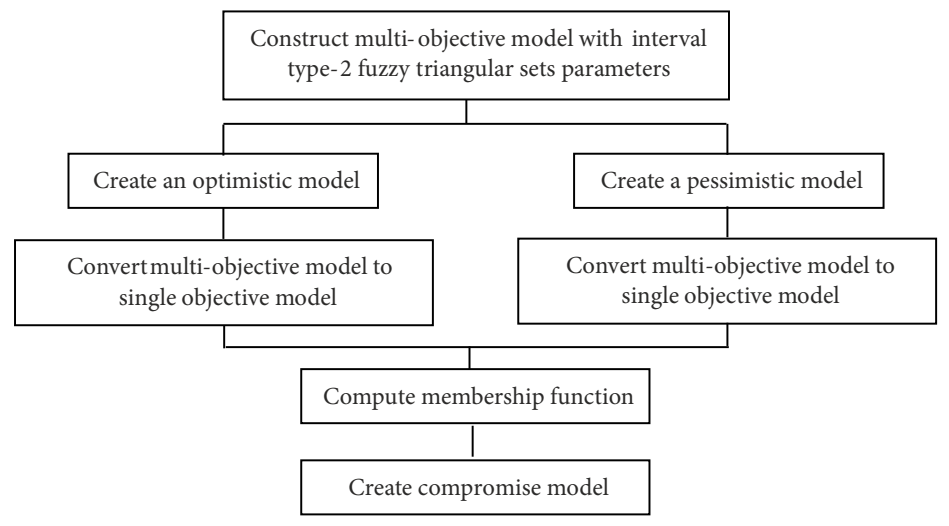

Figure 2. A flowchart of proposed solution procedure 


\section{Application examples}

Hiring a set of test problems, the solution approaches for the PPSS are examined. In the multi-project scheduling setting, the resource capacities are readily present. Multi-project problems are generated by combining 12,8 , and 5 projects with time horizons 15,10 , and 10 , respectively. The problem sets have three resources and two types of synergies. The proposed model is executed by GAMS optimization software.

- A set of parameters or characteristics can be considered while creating the instances.

- Number of objectives: We regard 3 objectives. For all three instances with maximizing expected profit and minimizing number of interruptions and tardiness functions.

- Duration of the candidate projects: they are defined by normalized IT2FT parameters.

- Planning horizon: it takes values 15, 10, and 10 time-periods for three examples, respectively.

- Resources: The levels of resources at hand in every time are determined by normalized normalized IT2FT parameters.

- Least projects: it involves the minimum number of candidates which can be active from subsets of projects $A j$ to fulfill the effect of linear synergy.

- Most projects: it takes the maximum number of candidates which can be active from subsets of projects $A j$ to fulfill the effect of linear synergy. This parameter takes the value corresponding to the number of $A j$ elements.

The remainder characteristics of the problem, e.g., resource expenditure, an increase or decrease in resource demand because of occurrence of synergy, expected profit, and resource cost, are determined by IT2FT-parameters.

The optimum results for three instances, entailing aggregated objective value, each of three objectives values, projects which are selected, and run time to the optimal solution are illustrated in Table 1.

Table 1. Brief results for different test problems

\begin{tabular}{|c|c|c|c|c|c|c|}
\hline $\begin{array}{c}\text { Number of } \\
\text { candidate projects }\end{array}$ & $\begin{array}{c}\text { Compromised } \\
\text { obj. }\end{array}$ & $\begin{array}{c}1 \text { st } \\
\text { obj. }\end{array}$ & $2 \mathrm{~d}$ obj. & $\begin{array}{c}3 \mathrm{~d} \\
\text { obj. }\end{array}$ & Selected projects & $\begin{array}{c}\text { CPU time } \\
(\mathrm{sec})\end{array}$ \\
\hline 5 & 0.453 & -14 & 2728.127 & 4 & $1,2,3,4$ & 14.113 \\
\hline 8 & 0.632 & -27 & 14756.813 & 8 & $1,2,3,4,5,6,7,8$ & 13.969 \\
\hline 12 & 0.522 & -37 & 15800.199 & 9 & $1,3,4,5,6,7,9,11,12$ & 135.146 \\
\hline
\end{tabular}

\section{Discussion of results}

In this part, four test problem scenarios are used to show the features of the presented model. A group of 8 nominated projects and 10 periods over the horizon $(T=10)$ can be investigated. All the projects which are selected ought to be planned throughout the accessible complete periods. Moreover, three kinds of resources needed for running projects could be accessible. There are two interdependent subsets for synergistic benefit in terms of resource consumption. There are dependencies between projects $2,3,4,6$, and 8 for synergy type 1 and between projects $1,2,3,5,6$, and 7 for synergy type 2 . 
The minimum and maximum number of running projects in each period to synergy occurrence are 2 and 6 for synergy type 1, 3, and 6 for synergy type 2 . Resource consumption, an increase or decrease in resource requirement due to the synergy occurrence, expected profit, and resource cost could be defined by crisp values for scenarios 1, 2, 3, and 4 and by normalized IT2FT-parameters for scenario 5. The total budget is equal to 2000 .

Scenario I: Project portfolio selection with resource constraints

A numerical instance that studies un-splitting PPSS, in which synergy and flexible due date do not happen, is provided in this sub-section. The single restriction is a scarcity of resources and limitation of budget. A single objective model with maximization expected profit is solved. The results are put forward in Table 2.

Table 2. The result of the first scenario

\begin{tabular}{|c|c|c|c|}
\hline \multirow{2}{*}{ Selected project } & \multirow{2}{*}{ Project scheduling } & Obj. & \multirow{2}{*}{ CPU time (sec) } \\
\cline { 3 - 3 } & & Expected Profit & \\
\hline 2 & $1,2,3,4,5,6,7,8$ & 2232.154 & 10.112 \\
\hline
\end{tabular}

Scenario II: Project portfolio selection with synergy

The assessment of the effect of interdependency among projects from the aspect of synergy on the PPSS is conducted via running this numerical example. A single objective model aiming at expected profit maximization is solved. According to their positive synergy, the number of resources required is less than that of the projects' resources in several periods. Thus, more projects (i.e., 2, 4, 6, and 7) have been chosen than the previous scenario. The results are reported in Table 3.

Table 3. The result of the second scenario

\begin{tabular}{|c|c|c|c|}
\hline \multirow{2}{*}{ Selected project } & Project scheduling & Obj. & \multirow{2}{*}{ CPU time (sec) } \\
& & Expected Profit & \\
\hline 2 & $1,2,3,4,5,6,7,8$ & & \multirow{2}{*}{10.87} \\
\hline 4 & $1,2,3,4,5,6$ & \multirow{2}{*}{10237.753} & \\
\hline 6 & $1,2,3,4,5,6,7,8$ & & \\
\hline 7 & $1,2,3,4,5,6,7$ & & \\
\hline
\end{tabular}

Scenario III: Project portfolio selection with synergy and projects' splitting

This sub-section provides a numerical instance to study an optimization approach regarding an allowance of splitting and synergies between projects. Actually, the allocation of more resources to candidates is not needed, and candidates are just planned with the point of covering accessible assets throughout a period. To handle it, a bi-objective model aiming at joint maximization of the total profit and minimization of the candidates' interruption is considered. Compared to the previous scenario, a better output is expected because the splitting compensates for the lack of resource availability. Splitting assumption increases the expected profit. Table 4 indicates the computational results. 
Table 4. The result of the third scenario

\begin{tabular}{|c|c|c|c|c|}
\hline \multirow{2}{*}{ Selected project } & \multirow{2}{*}{ Project scheduling } & \multicolumn{2}{|c|}{ Obj. } & \multirow{2}{*}{$\begin{array}{l}\text { CPU time } \\
\quad(\mathrm{sec})\end{array}$} \\
\hline & & Expected Profit & Projects' Interruption & \\
\hline 2 & $2,3,4,5,6,7,8,9$ & 12870.772 & 8 & \multirow{5}{*}{15.465} \\
\hline 3 & $1,3,4,5,6,7,8,9$ & \multirow{2}{*}{\multicolumn{2}{|c|}{ Aggregated Obj. }} & \\
\hline 4 & $1,2,3,4,5,6$ & & & \\
\hline 5 & $1,2,5,6,7,8,9$ & \multirow{2}{*}{\multicolumn{2}{|c|}{0.555}} & \\
\hline 7 & $1,2,3,4,7,8,9$ & & & \\
\hline
\end{tabular}

Scenario IV: Project portfolio selection with synergy, flexible due date and projects' splitting

This part proposes a multi-objective model focusing on the expansion of all-out anticipated benefit, minimization of the summation of the total measure of interruption in conducting the chose undertakings, and minimization of delay value. Candidates can be stopped, and in the wake of being part, these could run again in some other periods. Moreover, projects are chosen and planned as their due date constraints. Flexible due date provides more chance for projects to be implemented lateness, with paying the corresponding penalty. Table 5 illustrates the results of this example.

Table 5. The result of the fourth scenario

\begin{tabular}{|c|c|c|c|c|c|}
\hline \multirow{2}{*}{$\begin{array}{l}\text { Selected } \\
\text { project }\end{array}$} & \multirow{2}{*}{ Project scheduling } & \multicolumn{3}{|c|}{ Obj. } & \multirow{2}{*}{$\begin{array}{l}\text { CPU time } \\
\quad(\mathrm{sec})\end{array}$} \\
\hline & & Expected Profit & Projects' Interruption & Due date & \\
\hline 2 & $1,2,4,5,6,7,8,9$ & 12325.535 & 8 & -1 & \multirow{5}{*}{20.858} \\
\hline 4 & $1,2,3,4,5,6$ & \multirow{2}{*}{\multicolumn{3}{|c|}{ Aggregated Obj. }} & \\
\hline 5 & $1,2,3,6,7,8,9$ & & & & \\
\hline 6 & $1,2,3,4,5,7,8,9$ & \multirow{2}{*}{\multicolumn{3}{|c|}{0.565}} & \\
\hline 7 & $3,4,5,6,7,8,9$ & & & & \\
\hline
\end{tabular}

Scenario V: Project portfolio selection with synergy, flexible due date and projects' splitting in the presence of IT2FT-parameters

This sub-section provides an example based on the former scenario with the difference that parameters take IT2FT-numbers. The outcomes of handling this multi-objective approach can be reported in Table 6.

The aim of this section is to elaborate on the influence of each characteristic of the proposed model. All characteristics are noticed by the aim of constructing a model close to the real situations. In the real-world, some set of projects have overlapping that performing together lead to decrease resource requirement. Considering this feature can make more revenue by selecting more projects that has been certified by scenario II. On the other hand, the resources are not available unlimited. The splitting assumption not only reduces the need of resources but also improves the total benefit. Even though splitting can make better portfolio, the importance of projects' due dates cannot be neglected. Despite the fact 
Table 6. The result of the fifth scenario

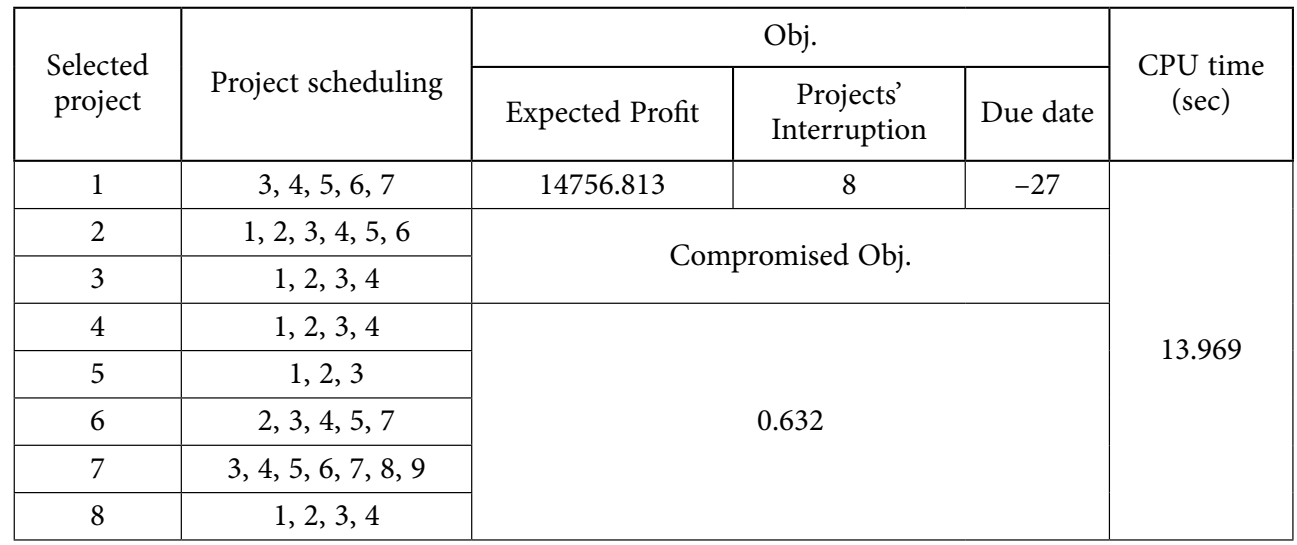

that the overall objective has been improved in scenario IV compared with scenario III, the total benefit has been deteriorated. The tardiness minimization objective prevents the high interruption and delays in project completion. All four scenarios are solved based on crisp values. In reality, the ever-changing and highly uncertain environment of projects make it difficult to estimate the parameters. Consequently, unreal data can be misleading in project selection and scheduling. Scenario V uses IT2FT-parameters and improves the aggregated objective and total profit.

\section{Conclusions}

In this paper, a new optimization model is developed for the PPSS problem by splitting over several intervals during the planning horizon and considering interdependency among projects in terms of synergy where selected projects can be implemented on the flexible due date. This study presents an optimization approach for the PPSS issue where two different concerns are jointly considered: how to select and how to schedule well-organized project portfolios. To handle the resources more efficient during the planning horizon, it is suggested to take into account these two interdependent procedures. These assumptions fill a gap in this field of study and provide lots of managerial insights. Our work contains some essential elements needed to make a proper selection: multiple conflicting objectives, unequal accessibility and consumption of resources, constraints of capital flow, and synergy among projects. In this paper, a new method is proposed to resolve linear programming issue with coefficients of the objective and constraints containing IT2F-numbers, and utilized it to deal with a PPSS issue with projects duration, availability of resource, cost of resource, resource expenditure, expected profit and synergy as proposed by IT2FT-numbers. The represented procedure is computationally well designed, and it can be hired to deal with this kind of decision concerns containing IT2F-parameters. Furthermore, a mixed-integer programming approach could be extended for the presented problem. The model aims at maximizing the firm's cash flow by taking into account the interest rate and minimizing the cost of tardiness 
and number of splitting. The outcomes of the presented approach with three sets of instances of various sizes are examined. The validity of model is evaluated through five different scenarios. Scenarios are initialized from the simplest assumption, single objective crisp model with resource constraints, and gradually progressed to proposed multi-objective model under IT2FT-parameters with flexible due date, synergy, and splitting assumptions. The results are discussed and illustrated the superiority of proposed model in terms of a number of selected projects, improving the value of objectives, and compromised objective. This study can be later developed to contain further detailed skill mapping of labor into the model to make sure that the deliverables' quality would be allowable. Other future study ideas would be taken into account time-dependent resource expenditure rates. The development of this model for activity setup times can be investigated. Other kinds of uncertainties in the projects may be considered in the modeling. Transfer time of resources between projects in the same class can be regarded. In the field of solution approach, more powerful strategies tackling the fuzziness and multi-objective model simultaneously can be interested.

\section{Acknowledgements}

The authors would like to thank anonymous referees for their valuable comments on the initial version of this paper.

\section{Author contributions}

S. Zolfaghari and S.M. Mousavi designed the research, analyzed the data and the obtained results and developed the paper. J. Antucheviciene provided extensive advice throughout the study regarding the research design, methodology and findings. All the authors have read and approved the final manuscript.

\section{Disclosure statement}

The authors declare that they have any competing financial, professional, or personal interests from other parties.

\section{References}

Baker, H. K., \& English, P. (2011). Capital budgeting valuation: financial analysis for today's investment projects. John Wiley \& Sons. https://doi.org/10.1002/9781118258422

Bhattacharyya, R., Kumar, P., \& Kar, S. (2011). Fuzzy R\&D portfolio selection of interdependent projects. Computers \& Mathematics with Applications, 62(10), 3857-3870. https://doi.org/10.1016/j.camwa.2011.09.036

Chen, J., \& Askin, R. G. (2009). Project selection, scheduling and resource allocation with time dependent returns. European Journal of Operational Research, 193(1), 23-34. https://doi.org/10.1016/j.ejor.2007.10.040 
Damghani, K. K., Sadi-Nezhad, S., \& Aryanezhad, M. B. (2011). A modular Decision Support System for optimum investment selection in presence of uncertainty: Combination of fuzzy mathematical programming and fuzzy rule based system. Expert Systems with Applications, 38(1), 824-834. https://doi.org/10.1016/j.eswa.2010.07.040

Dash, B., Narendran, T. T., \& Gajanand, M. S. (2016). A model for new product introduction under resource constraints and product interdependence. International Journal of Operational Research, 26(4), pp.473-508. https://doi.org/10.1504/IJOR.2016.077685

Dixit, V. and Tiwari, M.K., 2020. Project portfolio selection and scheduling optimization based on risk measure: a conditional value at risk approach. Annals of Operations Research, 285(1-2), 9-33. https://doi.org/10.1007/s10479-019-03214-1

Dorfeshan, Y., Mousavi, S. M., Vahdani, B., \& Siadat, A. (2019). Determining project characteristics and critical path by a new approach based on modified NWRT method and risk assessment under an interval type-2 fuzzy environment. Scientia Iranica, 26(4), 2579-2600.

https://doi.org/10.24200/sci.2018.50091.1503

Egri, P., \& Kis, T. (2020). Allocating raw materials to competing projects. Computers \& Industrial Engineering, 143, 106386. https://doi.org/10.1016/j.cie.2020.106386

Eshghi, A., Mousavi, S. M., \& Mohagheghi, V. (2019). A new interval type-2 fuzzy approach for analyzing and monitoring the performance of megaprojects based on earned value analysis (with a case study). Neural Computing and Applications, 31(9), 5109-5133. https://doi.org/10.1007/s00521-018-04002-x

Fox, G. E., Baker, N. R., \& Bryant, J. L. (1984). Economic models for R and D project selection in the presence of project interactions. Management Science, 30(7), 890-902. https://doi.org/10.1287/mnsc.30.7.890

Haghighi, M. H., Mousavi, S. M., \& Mohagheghi, V. (2019a). A new soft computing model based on linear assignment and linear programming technique for multidimensional analysis of preference with interval type-2 fuzzy sets. Applied Soft Computing, 77, 780-796.

https://doi.org/10.1016/j.asoc.2019.01.048

Haghighi, M. H., Mousavi, S. M., Antucheviciene, J., \& Mohagheghi, V. (2019b). A new analytical methodology to handle time-cost trade-off problem with considering quality loss cost under intervalvalued fuzzy uncertainty, Technological and Economic Development of Economy, 25(2), 277-299. https://doi.org/10.3846/tede.2019.8422

Hall, N. G., Long, D. Z., Qi, J., \& Sim, M. (2015). Managing underperformance risk in project portfolio selection. Operations Research, 63(3), 660-675. https://doi.org/10.1287/opre.2015.1382

Huang, X., \& Zhao, T. (2014). Project selection and scheduling with uncertain net income and investment cost. Applied Mathematics and Computation, 247, 61-71.

https://doi.org/10.1016/j.amc.2014.08.082

Javanmard, M., \& Nehi, H. M. (2019). A solving method for fuzzy linear programming problem with interval Type-2 fuzzy numbers. International Journal of Fuzzy Systems, 21(3), 882-891. https://doi.org/10.1007/s40815-018-0591-3

Kundu, P., Majumder, S., Kar, S., \& Maiti, M. (2019). A method to solve linear programming problem with interval type-2 fuzzy parameters. Fuzzy Optimization and Decision Making, 18(1), 103-130. https://doi.org/10.1007/s10700-018-9287-2

Li, X., Fang, S. C., Guo, X., Deng, Z., \& Qi, J. (2016). An extended model for project portfolio selection with project divisibility and interdependency. Journal of Systems Science and Systems Engineering, 25(1), 119-138. https://doi.org/10.1007/s11518-015-5281-1

Li, X., Fang, S. C., Tian, Y., \& Guo, X. (2015). Expanded model of the project portfolio selection problem with divisibility, time profile factors and cardinality constraints. Journal of the Operational Research Society, 66(7), 1132-1139. https://doi.org/10.1057/jors.2014.75 
Liao, S. H., \& Ho, S. H. (2010). Investment project valuation based on a fuzzy binomial approach. Information Sciences, 180(11), 2124-2133. https://doi.org/10.1016/j.ins.2010.02.012

Liesiö, J., Mild, P., \& Salo, A. (2008). Robust portfolio modeling with incomplete cost information and project interdependencies. European Journal of Operational Research, 190(3), 679-695. https://doi.org/10.1016/j.ejor.2007.06.049

Liu, S. S., \& Wang, C. J. (2011). Optimizing project selection and scheduling problems with timedependent resource constraints. Automation in Construction, 20(8), 1110-1119. https://doi.org/10.1016/j.autcon.2011.04.012

Mendel, J. M., John, R. I., \& Liu, F. (2006). Interval type-2 fuzzy logic systems made simple. IEEE Transactions on Fuzzy Systems, 14(6), 808-821. https://doi.org/10.1109/TFUZZ.2006.879986

Mirnezami, S. A., Mousavi, S. M., \& Mohagheghi, V. (2020). A new interval type-2 fuzzy approach for multi-scenario project cash flow assessment based on alternative queuing method and dependency structure matrix with a case study. Engineering Applications of Artificial Intelligence, 95, 103815. https://doi.org/10.1016/j.engappai.2020.103815

Mohagheghi, V., Mousavi, S. M., Antuchevičienė, J., \& Dorfeshan, Y. (2019). Sustainable infrastructure project selection by a new group decision-making framework introducing MORAS method in an interval type 2 fuzzy environment. International Journal of Strategic Property Management, 23(6), 390-404. https://doi.org/10.3846/ijspm.2019.10536

Mohagheghi, V., Mousavi, S. M., \& Vahdani, B. (2015). A new optimization model for project portfolio selection under interval-valued fuzzy environment. Arabian Journal for Science and Engineering, 40(11), 3351-3361. https://doi.org/10.1007/s13369-015-1779-6

Mohagheghi, V., Mousavi, S. M., Mojtahedi, M., \& Newton, S. (2020). Evaluating large, high-technology project portfolios using a novel interval-valued Pythagorean fuzzy set framework: An automated crane project case study. Expert Systems with Applications, 162, 113-117. https://doi.org/10.1016/j.eswa.2019.113007

Nowak, M., \& Trzaskalik, T. (2021). A trade-off multiobjective dynamic programming procedure and its application to project portfolio selection. Annals of Operations Research. https://doi.org/10.1007/s10479-020-03907-y

Peng, X., \& Huang, H. (2020). Fuzzy decision making method based on CoCoSo with critic for financial risk evaluation. Technological and Economic Development of Economys. https://doi.org/10.3846/tede.2020.11920

Rahman, H. F., Chakrabortty, R. K., \& Ryan, M. J. (2020). Memetic algorithm for solving resource constrained project scheduling problems. Automation in Construction, 111, 103052. https://doi.org/10.1016/j.autcon.2019.103052

Relich, M. (2021). A Decision Support System for Portfolio Management of NPD Projects. In decision support for product development (pp. 81-94). Springer, Cham.

https://doi.org/10.1007/978-3-030-43897-5_4

Salehi, K. (2018). Fuzzy multi-objective project selection problem using additive weighted fuzzy programming. Industrial Engineering Frontiers, 1(1), 1-15.

Santhanam, R., \& Kyparisis, G. J. (1996). A decision model for interdependent information system project selection. European Journal of Operational Research, 89(2), 380-399. https://doi.org/10.1016/0377-2217(94)00257-6

Selim, H., \& Ozkarahan, I. (2008). A supply chain distribution network design model: an interactive fuzzy goal programming-based solution approach. The International Journal of Advanced Manufacturing Technology, 36(3-4), 401-418. https://doi.org/10.1007/s00170-006-0842-6

Shafahi, A., \& Haghani, A. (2013, February). A linearization approach for project selection with interdependencies in resource costs. In ICORES (pp. 230-235). https://doi.org/10.5220/0004214402300235 
Tao, X., \& Schonfeld, P. (2006). Selection and scheduling of interdependent transportation projects with island models. Transportation Research Record, 1981(1), 133-141. https://doi.org/10.1177/0361198106198100120

Tofighian, A. A., \& Naderi, B. (2015). Modeling and solving the project selection and scheduling. Computers \& Industrial Engineering, 83, 30-38. https://doi.org/10.1016/j.cie.2015.01.012

Wang, J., \& Hwang, W. L. (2007). A fuzzy set approach for R\&D portfolio selection using a real options valuation model. Omega, 35(3), 247-257. https://doi.org/10.1016/j.omega.2005.06.002

Watermeyer, K., \& Zimmermann, J. (2020). A branch-and-bound procedure for the resource-constrained project scheduling problem with partially renewable resources and general temporal constraints. OR Spectrum, 42(2), 427-460. https://doi.org/10.1007/s00291-020-00583-z

Zhang, X., Hipel, K. W., \& Tan, Y. (2019). Project portfolio selection and scheduling under a fuzzy environment. Memetic Computing, 11(4), 391-406. https://doi.org/10.1007/s12293-019-00282-5 